\title{
Network meta-analysis comparing neoadjuvant chemoradiation, neoadjuvant chemotherapy and upfront surgery in patients with resectable, borderline resectable, and locally advanced pancreatic ductal adenocarcinoma
}

\author{
Qiancheng $\mathrm{Hu}^{1+}$, Dan Wang ${ }^{2+}$, Ye Chen ${ }^{1}$, Xiaofen $\mathrm{Li}^{1}$, Peng Cao ${ }^{1}$ and Dan Cao ${ }^{1 *}$ (D)
}

\begin{abstract}
Purpose: Neoadjuvant chemoradiation or chemotherapy has improved the treatment efficacy of patients with resectable, borderline resectable, and locally advanced pancreatic ductal adenocarcinoma (PDAC). Due to the optimal regimen remains inconclusive, we aimed to compare these treatments in terms of margin negative (R0) resection rate and overall survival (OS) with Bayesian analysis.
\end{abstract}

Patients and methods: We reviewed literature titles and abstracts comparing three treatment strategies (neoadjuvant chemoradiation, neoadjuvant chemotherapy, and upfront surgery) in PubMed, Embase, Cochrane Library, the American Society of Clinical Oncology and ClinicalTrials.gov database from 2009 to 2018 to estimate relative odds ratios (ORs) for margin negative (RO) resection rate and hazard ratios (HRs) for overall survival (OS) in all include trials.

Results: A total of 14 literatures with 1056 patients were enrolled in this Bayesian analysis. In the pairwise meta-analysis from limited head-to-head studies, compared with neoadjuvant chemotherapy, neoadjuvant chemoradiation showed superior OS significantly ( $\mathrm{HR} 0.8,95 \% \mathrm{Cl} 0.60-0.99, p<0.001)$ and there was no significant difference in $\mathrm{R} 0$ resection rate (OR 1.02, 95\%Cl 0.45-2.33, $P=34.6 \%$ ). However, in the network meta-analysis from all enrolled clinical trials, neoadjuvant chemoradiation showed significantly higher R0 resection rate over upfront surgery (HR 0.15, 95\% Crl 0.02-0.56), whereas neoadjuvant chemotherapy did not provide better efficacy in R0 resection over upfront surgery (HR 0.42, 95\% Crl 0.02-4.41) . For RO resection rate, neoadjuvant chemoradiation has the highest probability of ranking one compared with neoadjuvant chemotherapy or upfront surgery ( $79 \%$ vs $21 \%$ vs $0 \%$ ). For OS, neoadjuvant chemotherapy has the highest probability of ranking one compared with neoadjuvant chemoradiation or upfront surgery (98\% vs 0\% vs 2\%). Neoadjuvant chemotherapy was associated with higher rates of postoperative complications (rank worst: 84\%), followed by neoadjuvant chemoradiotherapy (13\%) and upfront surgery (3\%).

Conclusions: Different neoadjuvant treatment was selected based on various purposes, whether increasing R0 resection rate or not. Future clinical trials comparing neoadjuvant chemoradiation with neoadjuvant chemotherapy are warranted to confirm our results.

Keywords: Pancreatic ductal adenocarcinoma, Neoadjuvant therapy, Bayesian analysis, Network meta-analysis

\footnotetext{
* Correspondence: caodan316@163.com

${ }^{\dagger}$ Qiancheng Hu and Dan Wang are joint first authors

'Department of Abdominal Oncology, Cancer Center, West China Hospital,

Sichuan University, No. 37 Guo Xue Xiang, Chengdu 610041, China

Full list of author information is available at the end of the article
}

(c) The Author(s). 2019 Open Access This article is distributed under the terms of the Creative Commons Attribution 4.0 International License (http://creativecommons.org/licenses/by/4.0/), which permits unrestricted use, distribution, and reproduction in any medium, provided you give appropriate credit to the original author(s) and the source, provide a link to the Creative Commons license, and indicate if changes were made. The Creative Commons Public Domain Dedication waiver (http://creativecommons.org/publicdomain/zero/1.0/) applies to the data made available in this article, unless otherwise stated. 


\section{Introduction}

Pancreatic ductal adenocarcinoma (PDAC) is one of the most lethal cancers in the world [1]. Prognosis is dismal, and the 5-year survival rate is within 5\% [2]. Radical resection with a negative margin, such as margin-negative (R0) resection, is the key point for long-term survival of this aggressive malignancy [3].

Since 2009, local pancreatic ductal adenocarcinoma has been broadly classified into three categories: resectable, borderline resectable and locally advanced disease based on vascular involvement assessed by preoperative imaging in the expert consensus $[4,5]$. In general, approximately $10-20 \%$ of PDCA patients are present with surgically resectable disease [2], while $30-40 \%$ of patients are present with "borderline resectable pancreatic adenocarcinoma (BRPC)" or "locally advanced pancreatic adenocarcinoma (LAPC)". These patients have a low R0 resection rate and high potential of R1, making them theoretically the ideal candidates for neoadjuvant therapy [6].

It is becoming more obvious that patients with BRPC, who are at a higher risk for $\mathrm{R} 1$ resection, are potentially in need of neoadjuvant therapy with the goal of improving overall survival $(\mathrm{OS})[7,8]$. According to a systematic review and meta-analysis of 19 cohort studies, patients with unresectable disease (BRPC and LAPC) who had undergone neoadjuvant therapy had similar survival outcomes as the patients who were initially deemed resectable pancreatic adenocarcinoma (RPC) [9]. Dhir et al. evaluated the effects of neoadjuvant therapy including 5520 patients with local PDAC and reported that neoadjuvant therapy demonstrated OS benefit (HR $0.58,95 \%$ CI $0.46-0.70$ ), without increasing grade $\geq 3$ toxicities (OR $0.36,95 \% \mathrm{CI} 0.24-0.48$ ) [10]. Neoadjuvant therapy was also applied in managing patients with RPC sometimes, especially for patients with high-risk features. The systematic review confirmed that neoadjuvant treatment appeared to improve the OS by intention to treat, although showing lower overall resection rates for RPC or BRPC [11].

The studies mentioned above addressed the effectiveness and safety of neoadjuvant therapies involving neoadjuvant chemotherapy, or chemoradiation compared with upfront surgery in patients with local PDAC. However, they did not focus on the advantage between neoadjuvant chemoradiation and neoadjuvant chemotherapy, and there were very limited data available for head to head research comparing the main neoadjuvant regimens. Furthermore, to the best of our knowledge, no literature has reported a comprehensive comparison of three methods (neoadjuvant chemoradiation vs neoadjuvant chemotherapy vs upfront surgery). This study compared these methods for the treatment of resectable, borderline resectable, and locally advanced PDAC comprehensively by applying a network meta- analysis, with an expectation to provide some reference for selecting appropriate treatment.

\section{Methods \\ Search strategy and selection criteria}

The Bayesian analysis was conducted and reported in accordance with the PRISMA (Preferred Reporting Items for Systematic Reviews and Meta-Analyses) guidelines. We searched PubMed, Embase (Ovid), Cochrane Library, the American Society of Clinical Oncology and ClinicalTrials.gov database using "neoadjuvant therapies of pancreatic ductal adenocarcinoma" as part of the titles and abstracts with English restrictions. A time-frame from January 1, 2009, which was the date of introducing local anatomic subcategories of pancreatic ductal adenocarcinoma, i.e., RPC, BRPC and LAPC $[4,5]$, to December 16, 2018, was selected for the database search. In addition, we manually searched related reviews and bibliographies of included trials for additional references.

References were included if: 1. local PDAC; 2. compare two of the three treatment strategies (neoadjuvant chemoradiation, neoadjuvant chemotherapy, upfront surgery) with each other; 3 . report enough information to calculate hazard ratios (HRs); 4. unrestricted age, gender, performance status (PS), ethnicity and country.

The references were excluded according to the following criteria: 1 . neoadjuvant targeted therapy; 2 . neoadjuvant chemoradiation and chemotherapy mixed; 3 . posters and abstracts; 4. single-arm studies.

The search strategy in strict accordance with Population Intervention Comparison Outcomes Study (PICOS) design framework included the following domains of Medical Subject Heading (MeSH) terms: 'Pancreatic Neoplasms' and 'Neoadjuvant Therapy'; MeSH and Subheadings were combined with 'AND' or 'OR'.

\section{Data extraction and assessment for bias risk}

First, the titles and abstracts of articles were screened. Review articles, case series, case reports, guidelines and conference abstracts were excluded from our study; and then full-text articles that met the inclusion criteria were thoroughly reviewed. Two investigators (Qc H, D W) reviewed the full manuscripts independently and extracted information including patient characteristics, period and type of study, treatment protocols, the sample size and outcomes (median OS, hazard ratio, 95\% confidence interval and $\mathrm{R} 0$ resection rate) into the electronic database. Disagreements in study and data selection among investigators were resolved by discussion and consensus. The quality and risk of bias of randomized controlled trials (RCTs) were assessed by using Cochrane Collaboration's tool [12], and the other trials were assessed by Risk if Bias in Non-randomized Studies of Interventions (ROBINS-I) [13]. 


\section{Data synthesis and analysis}

The outcomes we analysed were median OS and R0 resection rate. R0 was defined as margin negative if tumour cells were present $>1 \mathrm{~mm}$ from the any surface. R1 was defined as margin positive if tumour cells were present within $1 \mathrm{~mm}$ from the any surface $[14,15]$. Results on OS in the Bayesian analysis were expressed as a hazard ratio (HR) with 95\% confidence intervals (CI). $P<0.05$ was considered as significant level. Heterogeneity was assessed with the $I^{2}$ statistic. $I^{2}$ values less than $25 \%$ and greater than $50 \%$ were regarded as indicating low and high heterogeneity, respectively [16]. When HRs were not reported, we made estimations from summary statistics with the method described by Tierney et al. in 2007 [17], and Kaplan-Meier curves were digitized using Getdata Graph Digitizer 2.26 (http://www.getdata-graphdigitizer.com). We applied the traditional pairwise metaanalysis between direct comparisons with Stata13 (StataCorp, College Station, TX, USA). The network metaanalysis was conducted with GeMTC version 0.14.3 (http://drugis.org/software/addis1/gemtc) and WinBUGS version1.4.3 (MRC Biostatistics Unit, Cambridge, UK) by fixed-effect models. For R0 resection rate, we used
GeMTC for network meta-analysis. Parameters for the GeMTC software were selected as: number of chains, 4; tuning iterations, 20,000; simulation iterations, 50,000; thinning interval, 10; inference samples, 10,000; and variance scaling factor, 2.5. Besides, we chose 5000 burnins and a thinning interval of 1 for each chain for HRs. The consistency model would be used when there was no significant inconsistency; otherwise, the inconsistency model was applied. We assessed the convergence of the model using the potential scale reduction factor (PSRF) of the Brooks-Gelman-Rubin method [18]; PSRF closer to 1 indicated the better convergence.

\section{Results}

\section{Eligible studies and characteristics}

We identified 1089 studies from the title and abstract review to start with (Fig. 1). After initial screening, we retrieved the full text of potentially eligible articles for further detailed assessment. With the predeveloped search strategy, 14 eligible publications including three randomized controlled trials were included for metaanalysis, with a total of 1056 patients received at least one of the three treatment strategies (Table 1) [19-32].

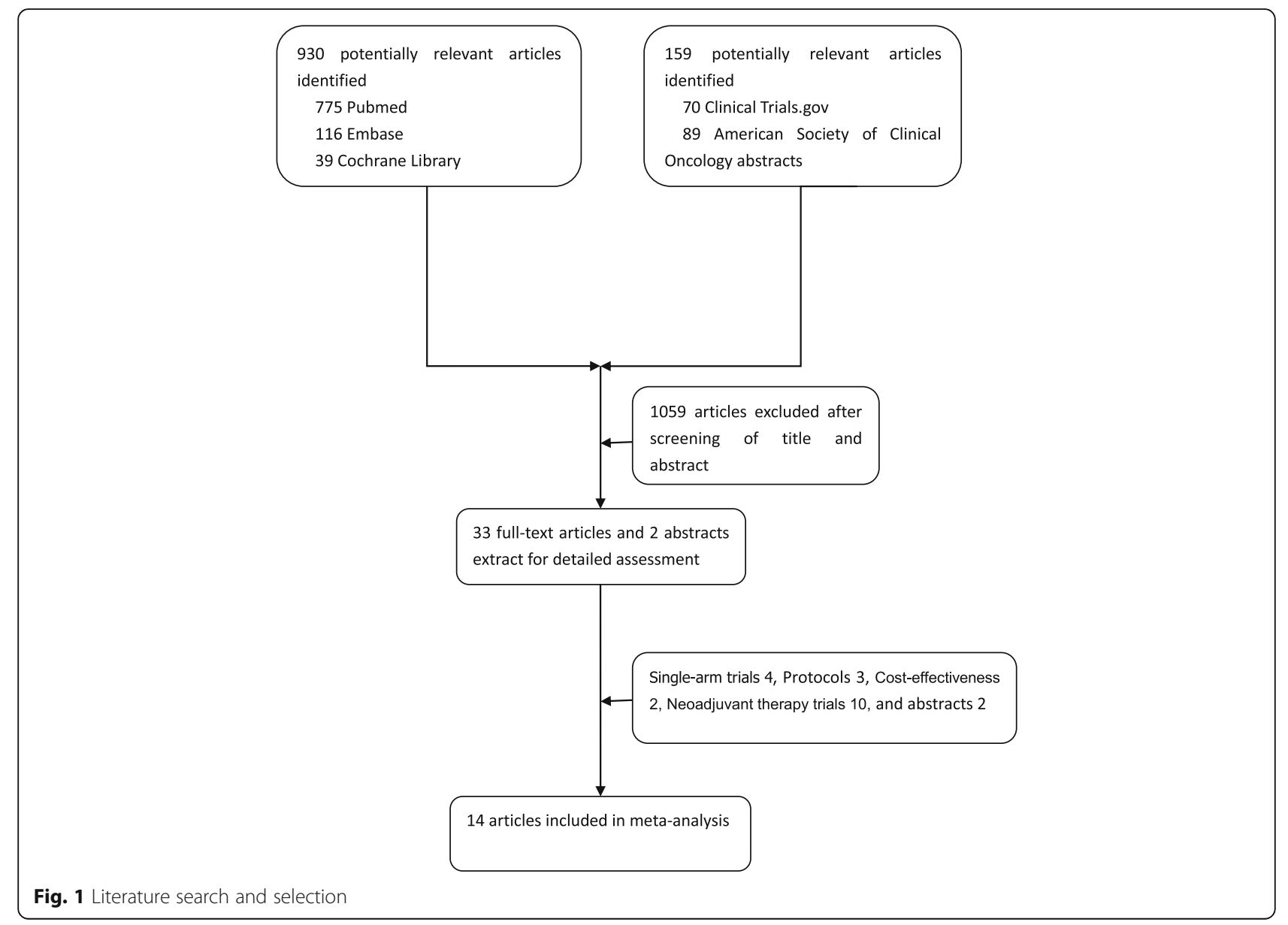


Table 1 Main characteristics of the controlled trials included in the meta-analysis

\begin{tabular}{|c|c|c|c|c|c|c|c|c|c|}
\hline References & Type of study & Disease & Period of study & Intervention regimen & Control regimen & Participants & Median OS & HR $(95 \% \mathrm{Cl})$ & RO (\%) \\
\hline $\begin{array}{l}\text { Hackert et } \\
\text { al. }\end{array}$ & Retrospective & LAPC & $2001-2015$ & Chemoradiotherapy & Chemotherapy & 322 vs 125 & 16.5 vs 16 & $0.93(0.67-1.28)$ & $31.3 \%$ vs $40.8 \%$ \\
\hline $\begin{array}{l}\text { Shrestha } \\
\text { et al. }\end{array}$ & Retrospective & BRPC & 2007-2012 & Chemoradiotherapy & Chemotherapy & 19 vs 14 & 16.4 vs 10.9 & $0.75(0.38-1.51)$ & NA \\
\hline Kim et al. & Retrospective & BRPC & $2007-2015$ & Chemoradiotherapy & Chemotherapy & 25 vs 15 & 20.1 vs 16.1 & $0.67(0.26-1.72)$ & NA \\
\hline Satoi et al. & Prospective & BRPC/LAPC & 2008-2013 & Chemoradiotherapy & Chemotherapy & 35 vs 32 & $\begin{array}{l}22 \text { vs Not } \\
\text { reached }\end{array}$ & $0.53(0.27-1.02)$ & $91 \%$ vs $81 \%$ \\
\hline $\begin{array}{l}\text { Lloyd et } \\
\text { al. }\end{array}$ & Retrospective & BRPC/LAPC & 2000-2013 & Chemoradiotherapy & Chemotherapy & 23 vs 65 & 12.5 vs 13.9 & $1.09(0.65-1.82)$ & $9 \%$ vs $6 \%$ \\
\hline $\begin{array}{l}\text { Barbier et } \\
\text { al. }\end{array}$ & Retrospective & LAPC & 1997-2006 & Chemoradiotherapy & Surgery-first & 88 vs 85 & 21.5 vs 18 & $1.16(0.7-1.91)$ & $92 \%$ vs $67 \%$ \\
\hline $\begin{array}{l}\text { Casadei et } \\
\text { al. }\end{array}$ & $\mathrm{RCT}$ & $\begin{array}{l}\text { RPC/BRPC/ } \\
\text { LAPC }\end{array}$ & $2007-2014$ & Chemoradiotherapy & Surgery-first & 18 vs 20 & 22.4 vs 19.5 & NA & $38.9 \%$ vs $25 \%$ \\
\hline $\begin{array}{l}\text { Casadei et } \\
\text { al. }\end{array}$ & Observtional & BRPC & $2000-2013$ & Chemoradiotherapy & Surgery-first & 30 vs 28 & NA & NA & 93.3 vs $71.4 \%$ \\
\hline Fujii et al. & Observtional & BRPC & $2002-2014$ & Chemoradiotherapy & Surgery-first & 21 vs 71 & 29.1 vs 13.1 & $0.28(0.10-0.75)$ & $100 \%$ vs $40 \%$ \\
\hline Fujii et al. & Prospective & $\mathrm{RPC} / \mathrm{BRPC}$ & $2001-2013$ & Chemoradiotherapy & Surgery-first & 40 vs 233 & 28.6 vs 33.7 & $0.79(0.28-2.22)$ & $86 \%$ vs $70 \%$ \\
\hline Jang et al. & $\mathrm{RCT}$ & BRPC & $2012-2014$ & Chemoradiotherapy & Surgery-first & 27 vs 23 & 21 vs 12 & $0.53(0.29-0.98)$ & $82.4 \%$ vs $33.3 \%$ \\
\hline $\begin{array}{l}\text { Tafima et } \\
\text { al. }\end{array}$ & Retrospective & RPC & $2006-2009$ & Chemotherapy & Surgery-first & 13 vs 21 & NA & $1.18(0.43-3.24)$ & $84.6 \%$ vs $85.7 \%$ \\
\hline $\begin{array}{l}\text { Golcher et } \\
\text { al. }\end{array}$ & $\mathrm{RCT}$ & $\mathrm{RPC}$ & $2003-2009$ & Chemoradiotherapy & Surgery-first & 33 vs 33 & 17.4 vs 14.4 & $0.96(0.55-1.67)$ & $89.5 \%$ vs $69.6 \%$ \\
\hline Murakami & Retrospective & BRPC & $2002-2015$ & Chemotherapy & Surgery-first & 52 vs 25 & 27.1 vs 11.6 & $0.42(0.25-0.72)$ & $72.3 \%$ vs $17.4 \%$ \\
\hline
\end{tabular}

et al.

a Data extraction from article; $R C T$ Randomised Controlled Trial, LAPC Locally Advanced Pancreatic Cancer, BRPC Borderline Resectable Pancreatic Cancer, RPC Resectable Pancreatic Cancer, NA Not answer

The eligible studies were published during 2010 to 2018. All studies included in our Bayesian analysis have been published as full manuscripts.

\section{Pairwise meta-analysis for $\mathrm{OS}$ and $\mathrm{R} 0$ resection rate}

There were five head-to-head studies which compared the OS between neoadjuvant chemoradiation and neoadjuvant chemotherapy. Within the five head-to-head studies, only three of them reported the R0 resection rate. In our pairwise meta-analysis, there was no significant difference between neoadjuvant chemoradiation and neoadjuvant chemotherapy in R0 resection rate (OR 1.02, 95\%CI 0.452.33, $I^{2}=34.6 \%$, Fig. 2a). Neoadjuvant chemoradiation showed superior OS significantly when compared with neoadjuvant chemotherapy (HR 0.8 , 95\% CI 0.60-0.99, $p<0.001$, Fig. 2b).

\section{Networks for multiple treatment comparisons}

The network was designed for three treatment comparisons of neoadjuvant chemoradiation, neoadjuvant chemotherapy and upfront surgery. All the potential scale reduction factors (PSRFs) were in the range of 1.00 to 1.01 , so the model was proven convergent and stable; the consistency model was adopted. According to the established network, based on the consistency model, neoadjuvant chemoradiation showed significantly higher R0 resection rate over upfront surgery (HR 0.15, 95\% CrI 0.02-0.56), whereas neoadjuvant chemotherapy did not provide better efficacy in R0 resection over upfront surgery (HR 0.42, 95\% CrI 0.02-4.41) (Fig. 3a). Compared with results from the traditional pairwise meta-analysis, neoadjuvant chemotherapy showed a superior advantage for OS over neoadjuvant chemoradiation (HR 0.72, 95\% CrI 0.58-0.88) or upfront surgery (HR 0.85, 95\% CrI $0.72-0.99$ ) in network meta-analysis (Fig. 3b).

The rank probabilities, calculated by the network consistency model, ranked the probabilities of rank order of treatment regimen overall outcomes evaluated (neoadjuvant chemoradiation, neoadjuvant chemotherapy and upfront surgery). Regimens with a higher value in the histogram were associated with higher probabilities for better treatment outcomes. The probability distribution of each regimen which was ranked at each of the possible positions was showed by the histogram (Fig. 4a-b). For the R0 resection rate, neoadjuvant chemoradiation had the highest probability of ranking one compared with neoadjuvant chemotherapy or upfront surgery (Fig. $4 \mathrm{a}, 79 \%$ vs $21 \%$ vs $0 \%$ ). For OS, neoadjuvant chemotherapy had the highest probability of ranking one compared with neoadjuvant chemoradiation or upfront surgery (Fig. 4b). Neoadjuvant chemoradiation ranked as highest ( $98 \%$ vs $0 \%$ vs $2 \%$ ) among all the regimens in all 
a 10

Hackert et al

Satoi et al

Lloyd et al

Overall (I-squared $=34.6 \%, p=0.217$ )

NOTE: Weghnts are trom random effects anaysis

b ID

Hackert et al

Shrestha et al

Kim et al

Satoi et al

Lloyd et al

Overall (I-squared $=0.0 \%, p=0.436$ )
OR $(95 \% \mathrm{Cl})$

Weight\%

$6.66(0.37,1.17) \quad 60.01$

$2.46(0.56,10.81) \quad 22.71$

$1.45(0.25,8.51) \quad 17.28$

$1.02(0.45,2.33) \quad 100.00$

$\underset{10.8}{1}$

HR $(95 \% \mathrm{Cl}) \quad$ Weight $\%$

$.93(0.67,1.28) \quad 41.68$

$0.75(0.38,1.51) \quad 12.15$

$0.67(0.26,1.72) \quad 7.28$

$0.53(0.27,1.02) \quad 27.57$

$1.09(0.65,1.82) \quad 11.33$

$0.80(0.60,0.99) \quad 100.00$

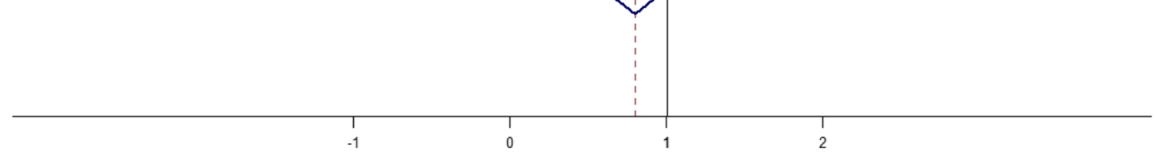

Fig. 2 a Comparison of RO resection rate according to pairwise meta-analysis. $\mathbf{b}$ Comparison of overall survival according to pairwise meta-analysis

\begin{tabular}{|l|l|l|}
\hline Surgergy-first & $\mathbf{0 . 4 2 ( 0 . 0 2 - 4 . 4 1 )}$ & $\mathbf{0 . 1 5}(\mathbf{0 . 0 2 - 0 . 5 6 )}$ \\
\hline Chemotherapy & $\mathbf{0 . 3 5 ( 0 . 0 5 - 2 . 6 )}$ \\
\hline $\mathbf{b}$ & chemoradiation \\
\hline Surgergy-first & $0.85(0.72,0.99)$ & $1.18(1.02,1.37)$ \\
\hline & Chemotherapy & $1.39(1.13,1.72)$ \\
\hline & & chemoradiation \\
\hline
\end{tabular}

Fig. 3 a Pooled odds ratios for Ro resection rate. (Red represents statistical significance). b Pooled hazard ratios for overall survival. (Red represents statistical significance) 

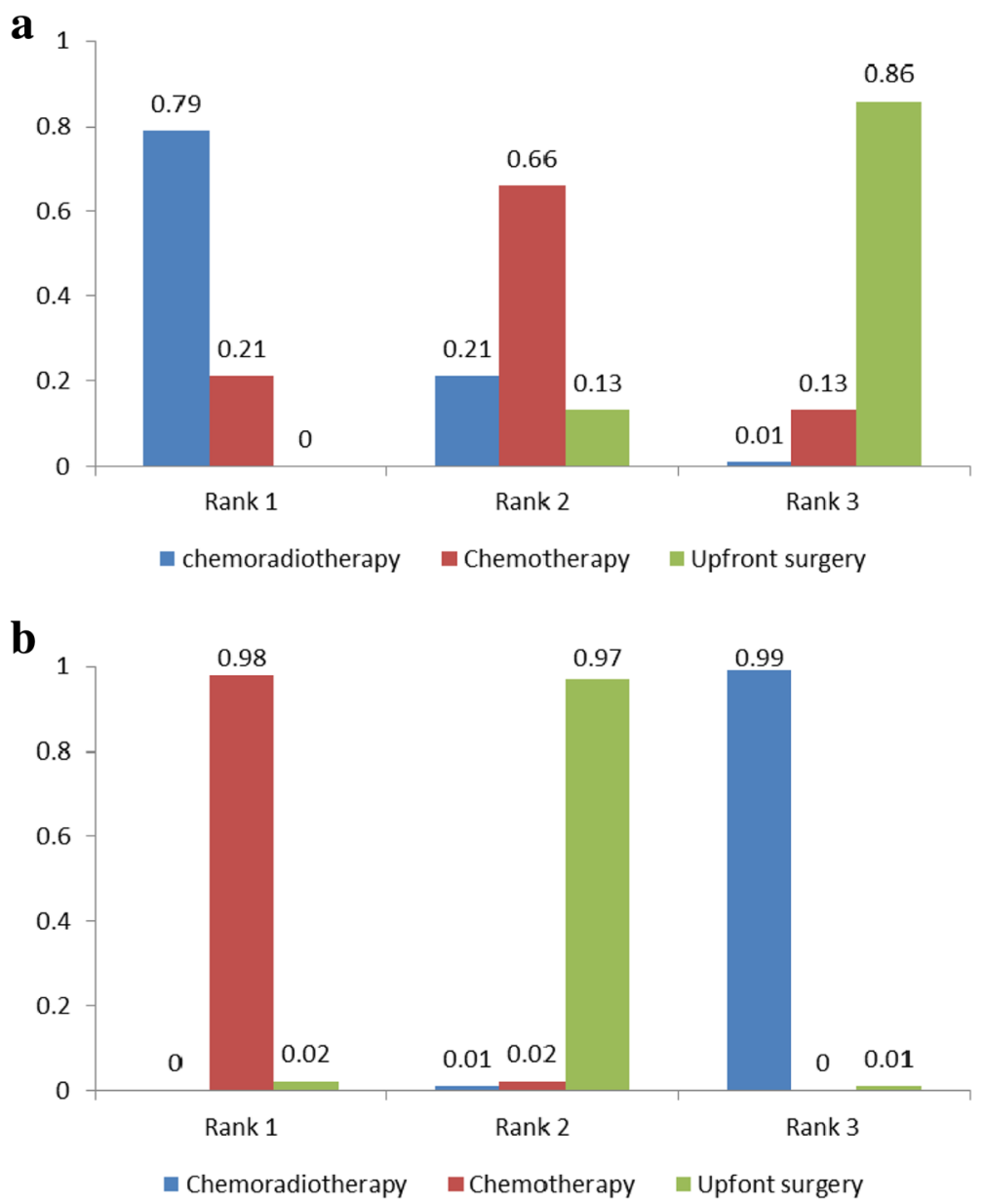

Fig. 4 Ranking of treatments in terms of Ro resection and overall survival

R0 resection rates measured without improving the likelihood of OS in local PDAC.

The comparison of postoperative complications among different treatment strategies was carried out. None of the five studies provided data on postoperative complications. The rankings of the three competitive treatment strategies were summarised in terms of postoperative complications (Fig. 5). Regimens with a higher value in the histogram were associated with higher probabilities for worse treatment outcomes. Neoadjuvant chemotherapy was associated with higher rates of postoperative complications (rank worst: $84 \%$ ), followed by neoadjuvant chemoradiotherapy (13\%) and upfront surgery (3\%).

\section{Discussion}

Our study compared neoadjuvant chemoradiation, neoadjuvant chemotherapy and upfront surgery for local PDAC including both R0 resection rate and OS by using pairwise meta-analysis and network meta-analysis. In our pairwise meta-analysis, there was no significant difference between neoadjuvant chemoradiation and neoadjuvant chemotherapy in R0 resection rate, with only three studies reporting the data. However, in this network analysis, our results suggested that neoadjuvant chemoradiation provided $\mathrm{R} 0$ resection rate advantage over upfront surgery in indirect evidence and had the highest probability of ranking one compared with neoadjuvant chemotherapy or upfront surgery. In general, neoadjuvant chemoradiation might be the most popular choice of treatment for local PDAC, especially for BRPC and LAPC.

In our study, neoadjuvant chemoradiation provided an OS advantage over neoadjuvant chemotherapy suggested by the pairwise meta-analysis, but not that of network meta-analysis likely because the enrolled patients could not be subgrouped, such as RPC, BRPC and LAPC. Zhan et al. proved that neoadjuvant therapy would be beneficial for patients with BRPC and LAPC in the meta-analysis of prospective studies, but neoadjuvant chemoradiation did not achieve a better prognosis than 


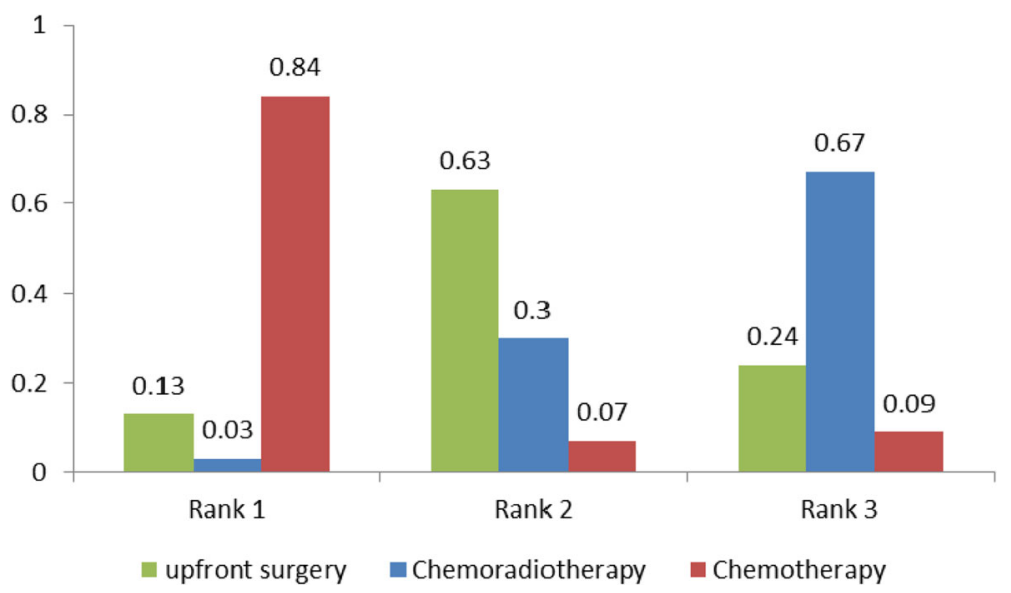

Fig. 5 Ranking of treatments in terms of postoperative complications

neoadjuvant chemotherapy alone (median OS: 16.7 months vs 16.8 months) [33]. As more than $75 \%$ of patients received neoadjuvant radiotherapy in the metaanalysis [34], controversies still existed, and it was difficult to achieve consensus on applying neoadjuvant chemoradiation and chemotherapy in the management of local PDAC.

Interestingly, Schorn et al. addressed that neoadjuvant therapy reduced the risk of local recurrence (RR 0.42, 95\%CI 0.32-0.55) but not the risk of distant metastasis (RR $1.02,95 \%$ CI $0.91-1.14$ ) in BRPC and LAPC in the metaanalysis of observational studies [34]. As the majority (91.7\%) of studies included neoadjuvant chemoradiation, it perhaps, be an effect of neoadjuvant chemoradiation on low local recurrence rate for local PDAC. This result illustrated neoadjuvant chemoradiation might be the most popular choice of treatment for local PDAC indirectly, and it was in accordance with our study. Subsequent therapies are another questionable factors for different results of direct and indirect meta-analysis [35].

Our study also had several limitations. First, although there was no obvious inconsistency and severe risk of bias detected, our study only enrolled three RCTs with a relatively small number of participants that might weaken the evidence of our meta-analysis. Second, given the dissimilar disease of participants with RPC, BRPC and LAPC, further subgroup analysis was not conducted. Lastly, different (neo) adjuvant chemotherapy regimens might had an effect on the OS.

\section{Conclusions}

In conclusion, according to the comprehensive evaluation suggested by Bayesian analysis, neoadjuvant chemoradiation provided better R0 resection rate advantage compared with neoadjuvant chemotherapy and upfront surgery, and neoadjuvant chemotherapy improved OS for local PDAC. Selection of different neoadjuvant therapy will reply on treatment purpose of whether need increase R0 resection rate or not. Additional head to head clinical data that further define the long-term efficacy of neoadjuvant chemoradiation and chemotherapy are needed to confirm our results.

\section{Abbreviations}

AEs: Adverse events; BRPC: Borderline resectable pancreatic adenocarcinoma; $\mathrm{Cl}$ : Confidence intervals; HRs: Hazard ratios; LAPC: Locally advanced pancreatic adenocarcinoma; MeSH: Medical Subject Heading; ORs: Relative odds ratios; OS: Overall survival; PDAC: Pancreatic ductal adenocarcinoma; PICOS: Population Intervention Comparison Outcomes Study Design; PRISMA: Preferred Reporting Items for Systematic Reviews and MetaAnalyses; PSRFs: Potential scale reduction factors; ROBINS-I: Risk Of Bias In Non-randomised Studies of Interventions; RPC: Resectable pancreatic adenocarcinoma

\section{Acknowledgements}

Thanks to Lu Guan (PhD, University of Victoria) for providing assistance and contributing to revisions.

\section{Authors' contributions}

Conceptualization: DC, QH, YC. Data curation: QH, DW. Methodology: QH, YC. Writing - original draft: $\mathrm{QH}, \mathrm{YC}, \mathrm{DC}, \mathrm{XL}, \mathrm{PC}$. All authors read and approved the final manuscript.

\section{Funding}

Not applicable.

\section{Availability of data and materials}

This Bayesian analysis used data from PubMed, Embase (Ovid), Cochrane Library, the American Society of Clinical Oncology and ClinicalTrials.gov database.

Ethics approval and consent to participate Not applicable.

Consent for publication

Not applicable.

\section{Competing interests}

The authors declare that they have no competing interests.

\section{Author details}

${ }^{1}$ Department of Abdominal Oncology, Cancer Center, West China Hospital, Sichuan University, No. 37 Guo Xue Xiang, Chengdu 610041, China. 
${ }^{2}$ Department of Medical Oncology, Cancer Center, West China Hospital, Sichuan University, Chengdu, China.

Received: 28 February 2019 Accepted: 27 June 2019

Published online: 10 July 2019

\section{References}

1. Siegel RL, Miller KD, Jemal A. Cancer statistics, 2017. CA Cancer J Clin. 2017; 67(1):7-30.

2. Malvezzi M, Carioli G, Bertuccio P, Boffetta P, et al. European cancer mortality predictions for the year 2017, with focus on lung cancer. Ann Oncol. 2017;28(5):1117-23.

3. Yamamoto $T$, Yagi S, Kinoshita $H$, Sakamoto $Y$, et al. Long-term survival after resection of pancreatic cancer: a single-center retrospective analysis. World J Gastroenterol. 2015:21(1):262-8.

4. Abrams RA, Lowy AM, O'Reilly EM, Wolff RA, et al. Combined modality treatment of resectable and borderline resectable pancreas cancer: expert consensus statement. Ann Surg Oncol. 2009;16(7):1751-6.

5. Callery MP, Chang KJ, Fishman EK, Talamonti MS, et al. Pretreatment assessment of resectable and borderline resectable pancreatic cancer: expert consensus statement. Ann Surg Oncol. 2009;16(7):1727-33.

6. Bockhorn M, Uzunoglu FG, Adham M, Imrie C, et al. Borderline resectable pancreatic cancer: a consensus statement by the international study Group of Pancreatic Surgery (ISGPS). Surgery. 2014;155(6):977-88.

7. Massucco P, Capussotti L, Magnino A, Sperti E, et al. Pancreatic resections after chemoradiotherapy for locally advanced ductal adenocarcinoma: analysis of perioperative outcome and survival. Ann Surg Oncol. 2006;13(9):1201-8.

8. Quiros RM, Brown KM, Hoffman JP. Neoadjuvant therapy in pancreatic cancer. Cancer Investig. 2007;25(4):267-73.

9. Laurence JM, Tran PD, Morarji K, Eslick GD, et al. A systematic review and metaanalysis of survival and surgical outcomes following neoadjuvant chemoradiotherapy for pancreatic cancer. J Gastrointest Surg. 2011;15(11):2059-69.

10. Dhir M, Malhotra GK, Sohal DPS, Hein NA, et al. Neoadjuvant treatment of pancreatic adenocarcinoma: a systematic review and meta-analysis of 5520 patients. World J Surg Oncol. 2017;15(1):183.

11. Versteijne E, Vogel JA, Besselink MG, Busch ORC, et al. Meta-analysis comparing upfront surgery with neoadjuvant treatment in patients with resectable or borderline resectable pancreatic cancer. Br J Surg. 2018;105(8):946-58.

12. Higgins JP, Altman DG, Gotzsche PC, Juni P, et al. The Cochrane Collaboration's tool for assessing risk of bias in randomised trials. BMJ. 2011;343:d5928.

13. Sterne JA, Hernan MA, Reeves BC, Savovic J, et al. ROBINS-I: a tool for assessing risk of bias in non-randomised studies of interventions. BMJ. 2016:355:14919.

14. Esposito I, Kleeff J, Bergmann F, Reiser C, et al. Most pancreatic cancer resections are R1 resections. Ann Surg Oncol. 2008;15(6):1651-60.

15. Verbeke CS, Leitch D, Menon KV, McMahon MJ, et al. Redefining the R1 resection in pancreatic cancer. Br J Surg. 2006;93(10):1232-7.

16. Mao L, Jian C, Changzhi L, Dan H, et al. Cytochrome CYP2C19 polymorphism and risk of adverse clinical events in clopidogrel-treated patients: a meta-analysis based on 23,035 subjects. Arch Cardiovasc Dis. 2013;106(10):517-27

17. Tierney JF, Stewart LA, Ghersi D, Burdett S, et al. Practical methods for incorporating summary time-to-event data into meta-analysis. Trials. 2007:8:16.

18. Yuan X, Liu WJ, Li B, Shen ZT, et al. A Bayesian network meta-analysis of whole brain radiotherapy and stereotactic radiotherapy for brain metastasis. Medicine (Baltimore). 2017;96(34):e7698.

19. Barbier L, Turrini O, Gregoire E, Viret F, et al. Pancreatic head resectable adenocarcinoma: preoperative chemoradiation improves local control but does not affect survival. HPB (Oxford). 2011;13(1):64-9.

20. Tajima H, Ohta T, Kitagawa H, Okamoto K, et al. Pilot study of neoadjuvant chemotherapy with gemcitabine and oral S-1 for resectable pancreatic cancer. Exp Ther Med. 2012;3(5):787-92.

21. Lloyd S, Chang BW. A comparison of three treatment strategies for locally advanced and borderline resectable pancreatic cancer. J Gastrointest Oncol. 2013;4(2):123-30.

22. Golcher H, Brunner TB, Witzigmann H, Marti L, et al. Neoadjuvant chemoradiation therapy with gemcitabine/cisplatin and surgery versus immediate surgery in resectable pancreatic cancer: results of the first prospective randomized phase II trial. Strahlenther Onkol. 2015;191(1):7-16.

23. Casadei R, Di Marco M, Ricci C, Santini D, et al. Neoadjuvant chemoradiotherapy and surgery versus surgery alone in resectable pancreatic cancer: a single-center prospective, randomized, controlled trial which failed to achieve accrual targets. J Gastrointest Surg. 2015;19(10):1802-12.

24. Lee JH, Kang CM, Bang SM, Choi JY, et al. The role of neoadjuvant Chemoradiation therapy in patients with borderline Resectable pancreatic Cancer with isolated venous vascular involvement. Medicine (Baltimore). 2015;94(31):e1233.

25. Fujii T, Yamada S, Murotani $K$, Kanda M, et al. Inverse probability of treatment weighting analysis of upfront surgery versus neoadjuvant chemoradiotherapy followed by surgery for pancreatic adenocarcinoma with arterial abutment. Medicine (Baltimore). 2015:94(39):e1647.

26. Fujii T, Satoi S, Yamada S, Murotani K, et al. Clinical benefits of neoadjuvant chemoradiotherapy for adenocarcinoma of the pancreatic head: an observational study using inverse probability of treatment weighting. J Gastroenterol. 2017;52(1):81-93.

27. Satoi S, Yanagimoto H, Yamamoto T, Ohe C, et al. Clinical outcomes of pancreatic ductal adenocarcinoma resection following neoadjuvant chemoradiation therapy vs. chemotherapy. Surg Today. 2017;47(1):84-91.

28. Hackert T, Sachsenmaier M, Hinz U, Schneider L, et al. Locally advanced pancreatic Cancer: neoadjuvant therapy with Folfirinox results in Resectability in 60\% of the patients. Ann Surg. 2016;264(3):457-63.

29. Murakami Y, Uemura K, Sudo T, Hashimoto Y, et al. Survival impact of neoadjuvant gemcitabine plus S-1 chemotherapy for patients with borderline resectable pancreatic carcinoma with arterial contact. Cancer Chemother Pharmacol. 2017;79(1):37-47.

30. Shrestha B, Sun Y, Faisal F, Kim V, et al. Long-term survival benefit of upfront chemotherapy in patients with newly diagnosed borderline resectable pancreatic cancer. Cancer Med. 2017:6(7):1552-62.

31. Kim HS, Jang JY, Han Y, Lee KB, et al. Survival outcome and prognostic factors of neoadjuvant treatment followed by resection for borderline resectable pancreatic cancer. Ann Surg Treat Res. 2017;93(4):186-94.

32. Jang JY, Han Y, Lee H, Kim SW, et al. Oncological benefits of neoadjuvant Chemoradiation with gemcitabine versus upfront surgery in patients with borderline Resectable pancreatic Cancer: a prospective, randomized, openlabel, multicenter phase 2/3 trial. Ann Surg. 2018;268(2):215-22.

33. Zhan $H X, X u J W, W u ~ D, W u ~ Z Y$, et al. Neoadjuvant therapy in pancreatic cancer: a systematic review and meta-analysis of prospective studies. Cancer Med. 2017;6(6):1201-19.

34. Schorn S, Demir IE, Samm N, Scheufele F, et al. Meta-analysis of the impact of neoadjuvant therapy on patterns of recurrence in pancreatic ductal adenocarcinoma. BJS Open. 2018;2(2):52-61.

35. Tempero MA, Malafa MP, Al-Hawary M, Asbun H, et al. Pancreatic adenocarcinoma, version 2.2017, NCCN clinical practice guidelines in oncology. J Natl Compr Cancer Netw. 2017;15(8):1028-61.

\section{Publisher's Note}

Springer Nature remains neutral with regard to jurisdictional claims in published maps and institutional affiliations.
Ready to submit your research? Choose BMC and benefit from:

- fast, convenient online submission

- thorough peer review by experienced researchers in your field

- rapid publication on acceptance

- support for research data, including large and complex data types

- gold Open Access which fosters wider collaboration and increased citations

- maximum visibility for your research: over $100 \mathrm{M}$ website views per year

At BMC, research is always in progress.

Learn more biomedcentral.com/submissions 